\title{
Methionine restriction on oxidative stress and immune response in dss-induced colitis mice
}

\author{
Gang Liu ${ }^{1}$, Lei Yu${ }^{2}$, Jun Fang ${ }^{3}$, Chien-An Andy $\mathrm{Hu}^{1,4}$, Jie Yin ${ }^{1}$, Hengjia $\mathrm{Ni}^{1}$, Wenkai \\ Ren $^{1}$, Veeramuthu Duraipandiyan ${ }^{5}$, Shuai Chen ${ }^{1}$, Naif Abdullah Al-Dhabi ${ }^{5}$ and \\ Yulong Yin ${ }^{1,6,7}$ \\ ${ }^{1}$ Key Laboratory of Agro-Ecological Processes in Subtropical Region, Institute of Subtropical Agriculture, Chinese Academy \\ of Sciences, Changsha 410125, China \\ ${ }^{2}$ China Animal Disease Control Center, Beijing 102618, China \\ ${ }^{3}$ College of Bioscience and Biotechnology, Hunan Agricultural University, Changsha 410128, China \\ ${ }^{4}$ Department of Biochemistry and Molecular Biology, University of New Mexico School of Medicine, Albuquerque, New Mexico \\ 87131, USA \\ ${ }^{5}$ Addiriyah Research Chair for Environmental Studies, Department of Botany and Microbiology, College of Science, King Saud \\ University, Riyadh 11451, Saudi Arabia \\ ${ }^{6}$ College of Animal Science, South China Agricultural University, Guangzhou 510642, China \\ ${ }^{7}$ Laboratory of Animal Nutrition and Human Health, School of Life Sciences, Hunan Normal University, Changsha 410081, \\ China \\ Correspondence to: Wenkai Ren, email: renwenkai19@126.com \\ Yulong Yin, email: yinyulong@isa.ac.cn \\ Keywords: inflammatory bowel disease, methionine restriction, diet, oxidative stress, NF- $\mathrm{K} B$ \\ Received: December 28, $2016 \quad$ Accepted: April 14, $2017 \quad$ Published: May 11, 2017 \\ Copyright: Liu et al. This is an open-access article distributed under the terms of the Creative Commons Attribution License 3.0 \\ (CC BY 3.0), which permits unrestricted use, distribution, and reproduction in any medium, provided the original author and source \\ are credited.
}

\section{ABSTRACT}

A strong correlation exists between inflammatory bowel disease (IBD) and oxidative stress involving alterations of several key signaling pathways. It is known that methionine promotes reactive oxygen species (ROS) production; we therefore hypothesize that a methionine restriction diet would reduce ROS production, inflammatory responses, and the course of IBD. We generated a murine colitis model by dextran sodium sulfate (DSS) treatment and tested the effects of the methionine restriction diet. Forty-eight mice were randomly divided into four groups of equal size, which included a control (CON) group, an MR (methionine restriction diet) group, a DSS treated group and an MR-DSS treated group. Mice in the first two groups had unrestricted access to water for one week. Mice in the two DSS-treated groups had unrestricted access to $5 \%$ DSS solution supplied in the drinking water for the same period. Mice in the CON and DSS groups were given a basal diet, whereas mice in the MR-DSS and MR groups were fed a $0.14 \%$ MR diet. We found that DSS reduced daily weight gain, suppressed antioxidant enzyme expression, increased histopathology scores and activated NF-KB and nuclear factor erythroid 2-related factor 2 / Kelch-like ECH-associated protein 1 (Nrf2/Keap1) signaling. We also showed that the MR diet upregulated catalase (CAT), superoxide dismutase (SOD), and glutathione peroxidase (GPX) activities, decreased myeloperoxidase (MPO), TNF-a and IL-1 $\beta$, and reversed activation of the NF-KB signaling pathway in MR-DSS mice. Taken together, our results imply that the MR diet may be considered as an adjuvant in IBD therapeutics. 


\section{INTRODUCTION}

Inflammatory bowel disease (IBD) is a chronic disorder of the gastrointestinal tract with increasing incidence around the world [1]. IBD, including ulcerative colitis (UC) and Crohn's disease, causes inflammation primarily affecting the gut mucosa and submucosa and is believed to be the result of a dysregulated immune response associated with environmental and genetic factors [2]. These factors include the microbiota, oxidative stress and diet [3, 4]. As part of the inflammatory process, the lamina propria of the gut is infiltrated by significant numbers of immune cells, which include eosinophils, polymorphonuclear neutrophils (PMNs) and activated macrophages. Once the lamina propria is infiltrated, the abovementioned cells generate a large quantity of reactive oxygen species (ROS) [5]. As ROS can cause lipid, protein and DNA oxidation and damage, it is believed that oxidative stress plays a critical role in the development of and/or the exacerbation of the symptoms of numerous diseases, including IBD [3]. In addition, the DNA transcription factor, nuclear factor- $\mathrm{kB}$ $(\mathrm{NF}-\mathrm{kB})$ and other gene expression regulators are crucially involved in the expression of pro-inflammatory cytokines, which are important for IBD initiation and pathogenesis [6]. An increasing use of immunomodulator and biologic therapies in the treatment of IBD has been reported.

Adverse effects and safety concerns limit the use of many pharmaceutical options (for example, antibody therapy, immune-modulators and steroids) for the treatment of IBD. Malignancy and infection are two of the major side effects $[7,8]$. The past two decades have witnessed the development of a number of experimental models designed to understand the pathophysiology and nutritional and pharmacological interventions of IBD in humans [9]. The major reason is that there are similarities in etiology, pathogenesis and therapeutic response between animal models and humans with IBD (particularly in UC). One of the commonly used methodologies is dextran sodium sulfate (DSS)-induced acute colitis [10], in which animals present an increased leukocyte recruitment response and production of cytokines and other inflammatory mediators, leading to tissue damage [11]. In addition, the expression of a number of genes (such as pro-inflammatory cytokines) important in the immune response, inflammation and oxidative damage are influenced by DSS treatment [12].

Interestingly, the use of methionine restriction (MR) diet increases the lifespan of a wide range of organisms, including yeast [13], fruit fly [14] and rodents [15], and decreases the onset, progression and incidence of several neurodegenerative conditions [16]. Furthermore, decreased methionine ingestion increases mitochondrial biogenesis [13] and reduces mitochondrial oxidative damage [17]. Moreover, methionine exhibits a regulatory effect on the electron transport chain of mitochondria, and when methionine is restricted from the diet, the amounts of mitochondrial complexes and ROS in rodents are significantly reduced [18]. However, whether an MR diet has any beneficial effect on IBD has not been investigated.

Previously, we reported the role of amino acids in nutritional regulation of the inflamed gut in animal models and demonstrated that some amino acids can be used as modulators of intestinal inflammatory diseases [19]. In this study, the impact of an MR diet on the immune response and oxidative stress was exploited in a mouse model of colitis.

\section{RESULTS}

As the most frequently used index in the clinic for IBD is weight loss, we monitored the mean daily weight gain following DSS treatment, and found that a significant decrease $(P<0.05)$ in the body weight was observed in the DSS group (Figure 1A). A general tendency to reduce this suppression in growth was seen in MR-IBD mice $(P>0.05)$ (Figure 1A). There was no discernible difference in colon length or colon weight between the four groups (Figure 1B and 1C). Significantly more severe colitis was observed in the colons from DSS-treated mice compared to the MR-DSS mice following the administration of DSS $(P<0.05)$ (Figure 2).

The extent of neutrophil infiltration in the mouse colonic tissue was quantified by measuring the activity of myeloperoxidase (MPO), as this enzyme is most abundant in the granulocytes of neutrophils. The mucosa was infiltrated by inflammatory cells, particularly neutrophils, in addition to the intestinal inflammation induced by the DSS. Table 1 shows the MPO and antioxidant enzyme activities. The MR diet had no impact on the MPO activity compared to the control group. A significantly $(P<0.05)$ increased MPO activity in the DSS-induced colitis was observed compared to the mice in the CON and MR group; this increase was suppressed by the administration of the MR diet $(P<0.05)$.

DSS administration resulted in a decrease in CAT activity, SOD activity, and GPx activity $(P<0.05)$, compared to the control and MR groups, but no significant changes in SOD, CAT, GRed or GPx were observed between the control and MR groups. Comparing the MR-DSS group with the DSS group, the former resulted in significantly higher SOD, CAT, and GPx activities $(P<0.05)$.

The 2,2'-azino-bis(3-ethylbenz-thiazoline-6-sulfonic acid) (ABTS) array was used to determine the antioxidant capacity in plasma. Figure 3 shows the impact of the administration of an MR diet on the plasma antioxidant power. There was a decrease in the DSS and MR-DSS groups compared with the MR and control groups $(P<$ $0.05)$. There was a significantly increased total antioxidant capacity in the MR-DSS group compared to the DSS group $(P<0.05)$, but no significant difference between the MR group compared to the CON group.

Figure 4 shows the colonic IL- $1 \beta$ and TNF- $\alpha$ levels of the four groups. The administration of the MR diet had no effects on the colonic level of the two compared to the CON group. However, there were significantly higher levels of both cytokines in the DSS and MR-DSS groups 
compared to both the CON and MR groups $(P<0.05)$. Both IL- $1 \beta$ and TNF- $\alpha$ levels were lower in the MR-DSS group that in the CON-DSS group $(P<0.05)$.
Measurements of nuclear NF- $\mathrm{B}$ (p65) were performed to evaluate NF- $\kappa \mathrm{B}$ activation in the colons of the mice. Treatment with DSS resulted in a significantly
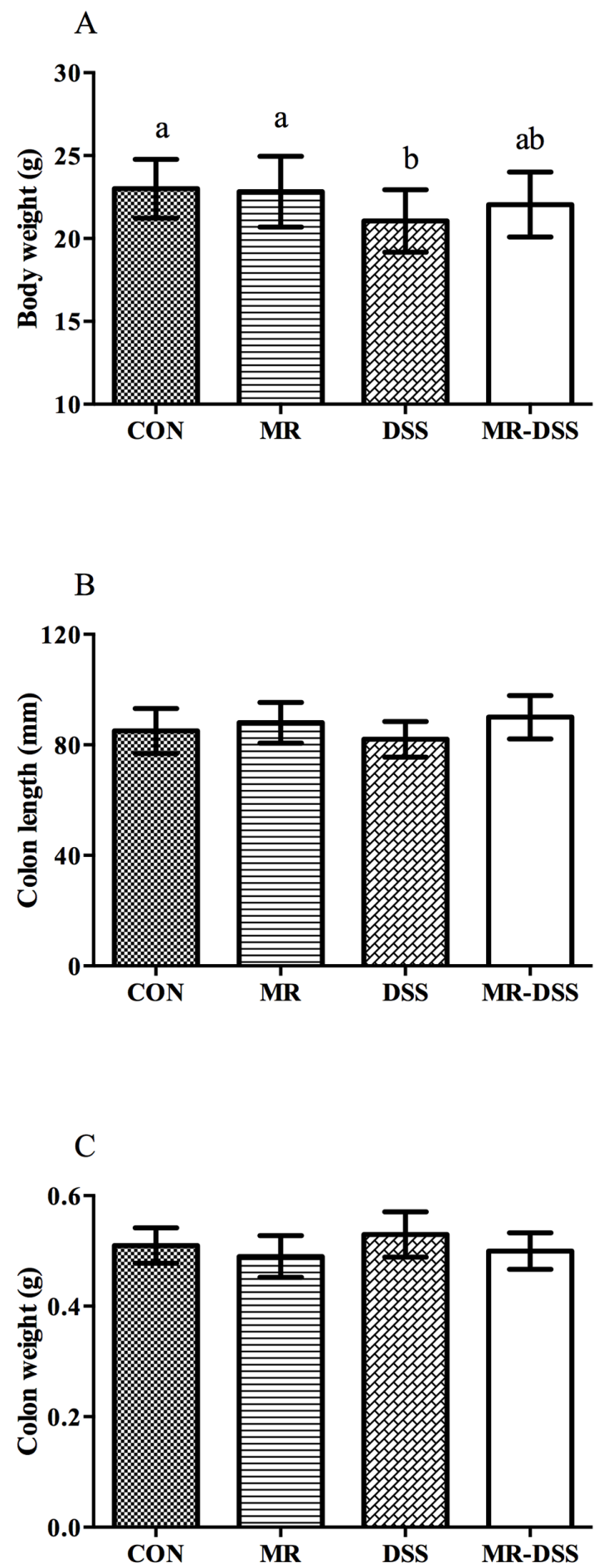

Figure 1: The effect of administration of an MR diet after DSS treatment on (A) body weight, (B) colon length and (C) colon weight. Data are presented as the mean \pm SEM. Values with different superscript letters were significantly different $(P<0.05$; $n=12$ ). 
Table 1: Colonic myeloperoxidase and antioxidant enzyme activities in mice following the induction of colitis by DSS administration

\begin{tabular}{lcccc}
\hline & CON (U/mg proteins) & DSS (U/mg proteins) & MR (U/mg proteins) & MR-DSS (U/mg proteins) \\
\hline MPO & $1.89 \pm 0.16^{\mathrm{a}}$ & $2.42 \pm 0.21^{\mathrm{b}}$ & $1.85 \pm 0.15^{\mathrm{a}}$ & $2.13 \pm 0.24^{\mathrm{c}}$ \\
SOD & $28.4 \pm 2.8^{\mathrm{a}}$ & $20.2 \pm 1.8^{\mathrm{b}}$ & $29.7 \pm 32.6^{\mathrm{a}}$ & $22.5 \pm 1.9^{\mathrm{c}}$ \\
CAT & $59.4 \pm 4.6^{\mathrm{a}}$ & $48.3 \pm 3.7^{\mathrm{b}}$ & $60.5 \pm 4.9^{\mathrm{a}}$ & $53.4 \pm 3.9^{\mathrm{c}}$ \\
GPx & $1.39 \pm 0.11^{\mathrm{a}}$ & $1.05 \pm 0.09^{\mathrm{b}}$ & $1.41 \pm 0.12^{\mathrm{a}}$ & $1.19 \pm 0.11^{\mathrm{c}}$ \\
GRed & $0.094 \pm 0.004$ & $0.097 \pm 0.003$ & $0.095 \pm 0.004$ & $1.004 \pm 0.003$ \\
\hline
\end{tabular}

Data are presented as the mean \pm SEM. Values with different superscript letters were significantly different.

activated nuclear NF- $\mathrm{kB}$ signal $(P<0.05)$, as shown in Figure 5A. No significant difference in nuclear NF- $\mathrm{KB}$ (p65) was seen between MR mice and the CON group. In the MR-DSS group, the activation was significantly suppressed by the MR diet although there was a significantly lower quantity of nuclear NF- $\mathrm{kB}$ in the MR mice than in the MR-DSS group $(P<0.05)$. For the Nrf2/ Keap1 signaling pathway, the MR diet had no impact on the Nrf2 signal compared to the control group as shown in Figure 5B, but there was clear enhancement of the colonic nuclear Nrf2 abundance resulting from DSS and MR-DSS groups compared to the control and MR groups $(P<0.05)$.

\section{DISCUSSION}

IBD in humans is associated with intermittent periods of acute gastrointestinal tract inflammation $[20,21]$. To investigate if an MR diet can reduce the symptoms of IBD, we selected a mouse colitis model that is induced by DSS. We found that $5 \%$ DSS induced acute colitis and reduced body weight in mice, which is consistent with previous work [10,22]. Evidence has shown the presence of high levels of MPO in IBD [23], and DSS increases colonic MPO activity through the induction of neutrophil infiltration, which is a marker of the inflammatory response within the colonic mucosa [24]. We observed a significant increase of MPO activity in the mice of the DSS and MR-DSS groups. As the migration of neutrophils and the increase of MPO activity are the main characteristics of chronic inflammation of the colon, our results validate the importance of an MR diet. In addition, inflammation is associated with the recruitment and activation of phagocytic leukocytes within the mucosa, which produce and release a large quantity of superoxide anion and other ROS [5] in an uncontrolled manner, potentially

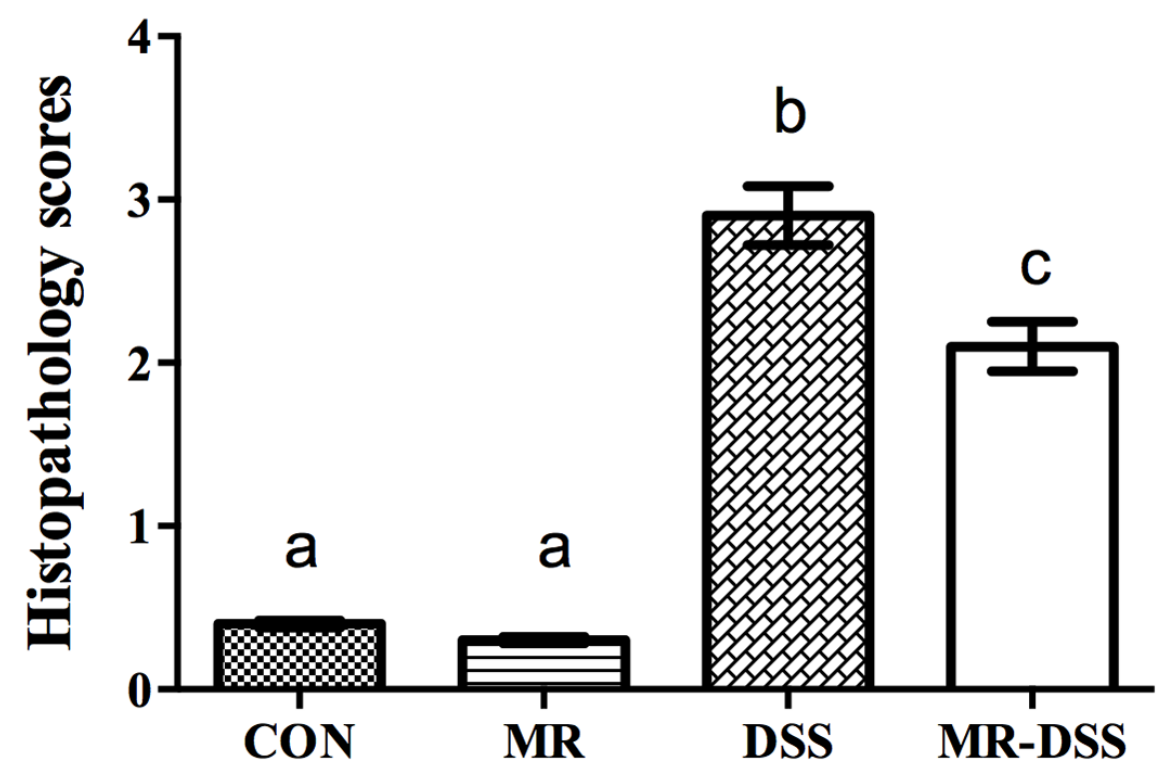

Figure 2: Histopathology scores given for mice in the control group and COS groups after 7 days of DSS administration. Data are presented as the mean \pm SEM. Values with different superscript letters were significantly different $(P<0.05 ; n=12)$. 


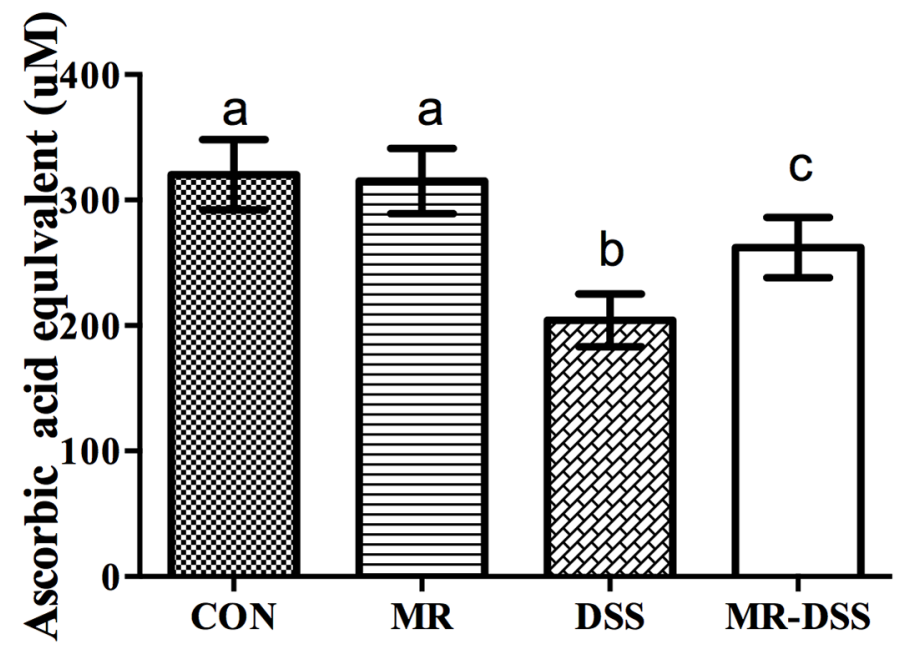

Figure 3: The effect of an MR diet on plasma antioxidant power. The total antioxidant capacity was measured in the mouse plasma treated as per the protocol using the ABTS assay. Data are presented as the mean \pm SEM. Values with different superscript letters were significantly different $(P<0.05 ; n=8)$.
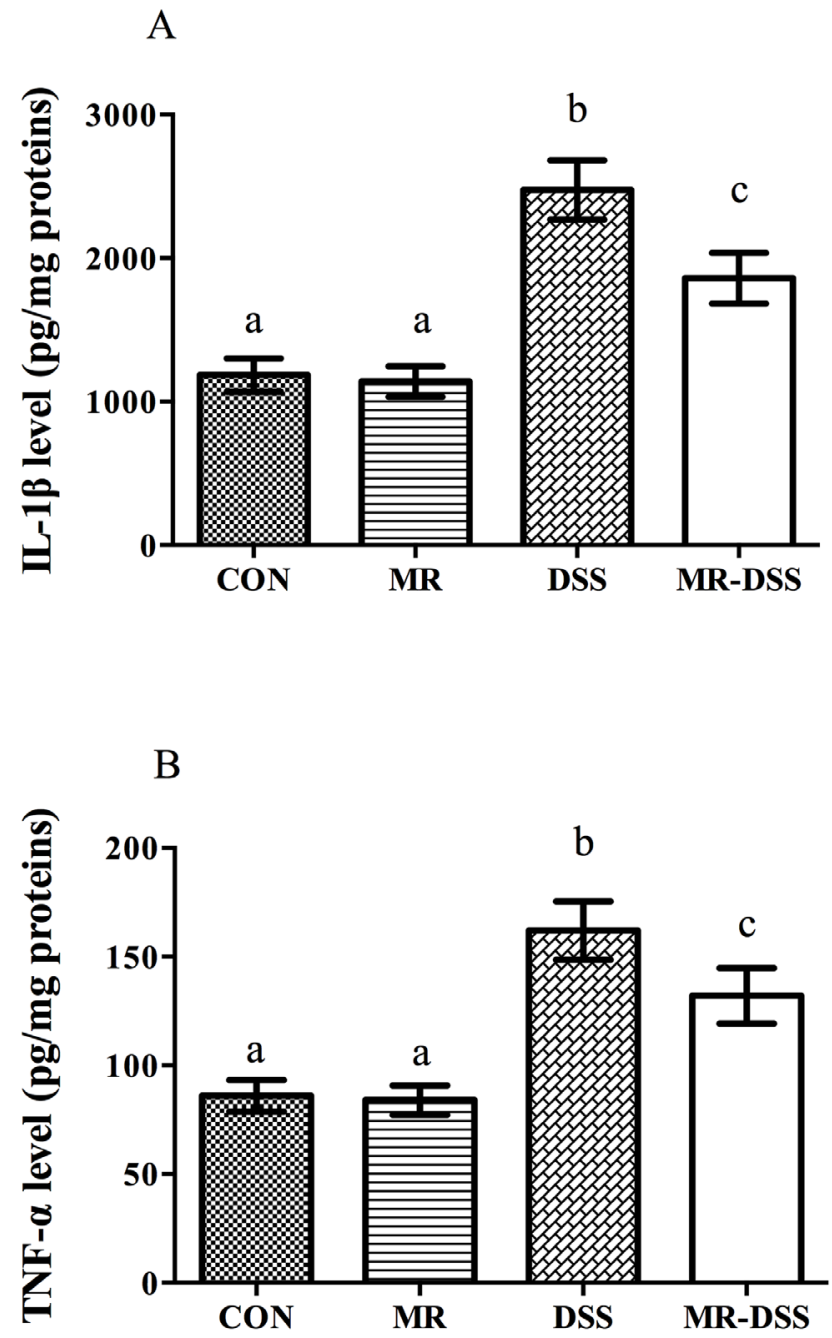

Figure 4: Colonic IL-1ß (A) and TNF- $\alpha$ (B) levels of four groups. Data are presented as the mean \pm SEM. Values with different superscript letters were significantly different $(P<0.05 ; n=8)$. 
causing cellular damage by overwhelming protective mechanisms [25]. Highly localized concentrations of ROS can attack and inactivate SOD and other antioxidant enzymes, preventing the neutralization of ROS. Thus, the significant reduction of SOD and CAT by DSS treatment contributes to the tissue damage, similar to the colitis phenotype in human IBD patients [26]. In fact, oxidative stress has two major effects: direct intestinal cell damage and initiation of inflammation through the activation of a number of signaling pathways, including NF- $\kappa \mathrm{B}$ [5]. This class of complex proteins is known [3] to be associated with the expression of a range of chemokines, adhesion factors and cytokines in response to stress (for example, oxidative stress and inflammation). The transcription of many pro-inflammatory genes, including those of adhesion molecules, chemokines, iNOS and inflammatory cytokines, can also be activated by NF- $\kappa$ B. In addition, our DSS-treated mice also showed an increase of excessive NF- $\kappa \mathrm{B}$, but when administered an MR diet, a significant suppression of NF- $\kappa \mathrm{B}$ activation, which is consistent with those in humans patients with IBD $[26,27]$. .

Beloqui et al. reported that pro-inflammatory cytokines are actively secreted in inflamed regions in IBD, indicating that pro-inflammatory cytokines are directly and heavily involved in immunopathogenesis [28]. Mice treated with DSS exhibited a significant upregulation of IL-1 $\beta$ expression compared to the healthy control group, demonstrating that colonic inflammation was induced by $5 \%$ DSS. Our results showed that mice treated with DSS, regardless of whether the MR diet was administered, exhibited upregulated levels of IL-1 $\beta$
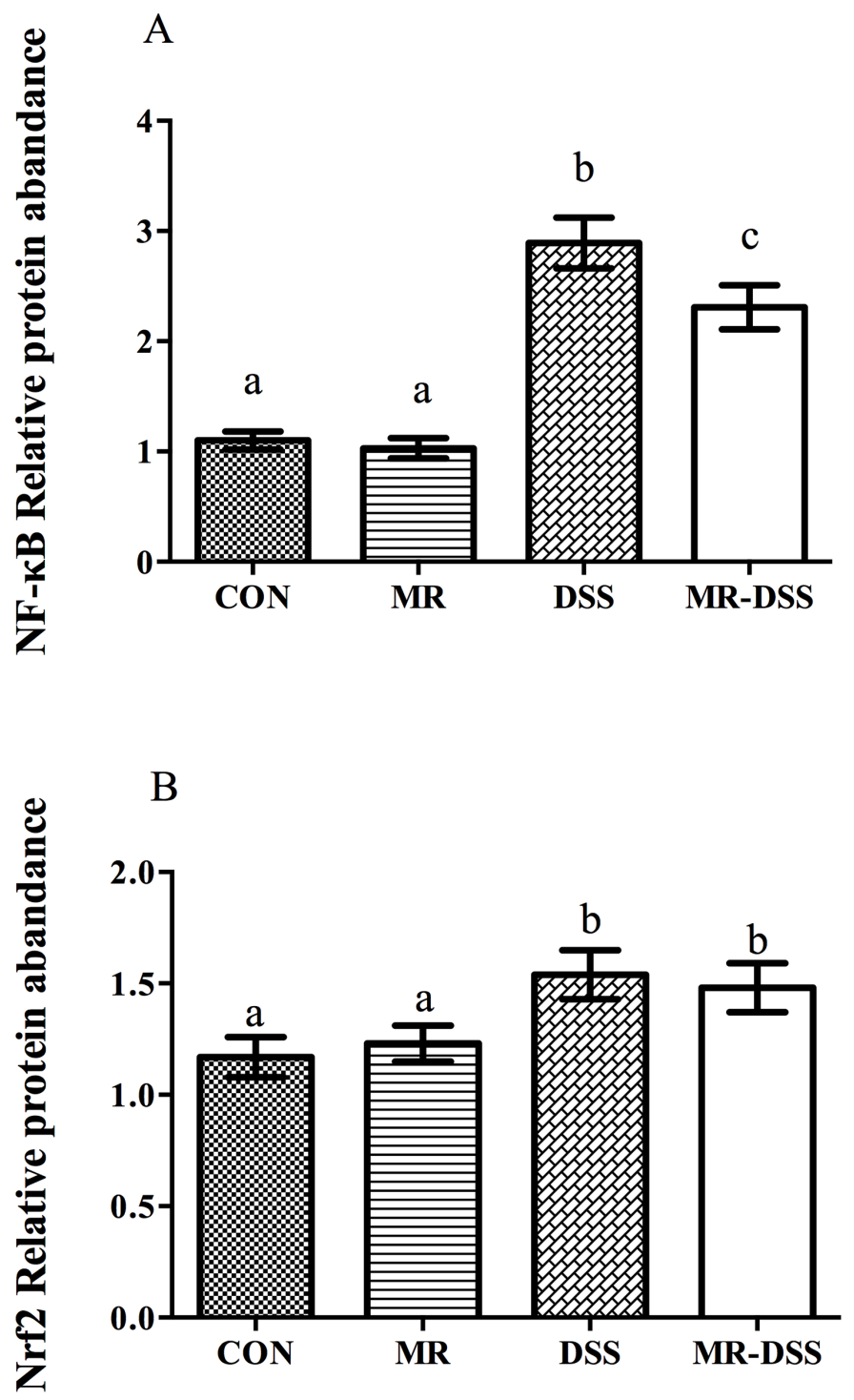

Figure 5: NF-кB (A) and Nrf2/Keap1 (B) signaling pathways in the DSS-induced colitis model. Data are presented as the mean $\pm \mathrm{SEM}$. Values with different superscript letters were significantly different $(P<0.05 ; n=8)$. 
and TNF- $\alpha$ in colon samples, which is consistent with patients with IBD [29] and other animal models of IBD. TNF- $\alpha$, a pleiotropic cytokine, is involved in numerous inflammatory processes relevant to IBD pathogenesis, including leukocyte recruitment, apoptosis, cytotoxic and phase response, intestinal permeability, and expression of adhesion molecules [30]. Thus, our results indicate that MR can be used as a potent immunomodulatory and anti-inflammatory modulator, and the MR diet should be considered as an adjuvant diet for animal models and human patients with IBD.

\section{MATERIALS AND METHODS}

\section{Animal and experimental design}

The animal work was performed in accordance with the Chinese Guidelines For Animal Welfare And Experimental Protocols and was approved by the Animal Care and Use Committee of the Institute of Subtropical Agriculture at the Chinese Academy of Sciences [31]. Forty male ICR mice, weighing approximately $21 \mathrm{~g}$, were kept in cages constructed of polycarbonate material with access to laboratory strip chow at all times. The temperature within the cages was controlled at $25^{\circ} \mathrm{C} \pm$ $3^{\circ} \mathrm{C}$, and the humidity was kept at $50 \pm 5 \%$. A 12 -hour cycle of light and dark was used. The mice were randomly divided into four groups of equal size randomly: a control group (CON), a group treated with DSS only (DSS), a group treated with MR (MR), and a group treated with MR and DSS (MR-DSS). The CON and MR groups were given free access to water for a period of seven days. Mice in the CON and DSS groups were given a basal diet (methionine $0.8 \%$ ) [19], and mice in the MR-DSS and MR groups were fed a $0.14 \%$ methionine MR diet. Each mouse in the DSS and MR-DSS groups were allowed seven days of free access to a $5 \%$ (wt/vol) DSS solution (molecular weight of 5,000 Dalton DSS (Kayon Biological Technology Co., Ltd.) dissolved in water), which was supplied to them as drinking water [19].

The morning of the eighth day, the average daily weight gain was calculated. Each mouse was then sacrificed, and the length and weight of the colon were measured and the colon tissue of each mouse was harvested and immediately frozen in liquid nitrogen for further analysis according to our published protocol [19].

\section{Histological analysis}

Colon tissue was fixed in $10 \%$ formalin. Hematoxylin and eosin staining of paraffin-embedded sections was performed. Six criteria were used to grade colitis histologically, including inflammation, edema, erosion, cryptitis, ulcers and goblet cell hyperplasia [32]. Lesions were scored using a scale of zero to four. Zero represented no colitis or epithelial thickening, one represented an increased number of mucosal leukocytes and/or slight epithelial cell hyperplasia, two represented multiple loci of inflammation, leukocytic mucosal and submucosal infiltration and/or marked epithelial cell hyperplasia (defined as a twofold to threefold increase in crypts), three represented extensive mucosal and submucosal leukocytes, depletion of mucin-secreting goblet cells and/or pronounced epithelial cell hyperplasia (defined as a threefold to tenfold increase in crypts), and four represented extensive transmural leukocytic infiltration, crypt abscesses and/or a marked epithelial cell hyperplasia (defined as a greater than tenfold increase in crypts).

\section{Determination of colonic antioxidant enzyme activities}

Protein concentrations were measured by a BCA assay kit (Pierce, Jiancheng Bioengineering Institute, Nanjing, China). Guaiacol oxidation by MPOproduced hydroxyl radicals in the presence of hydrogen peroxide was evaluated as previously described [33]. This activity, expressed in units per mg of protein, was defined as the quantity of enzyme degrading $1 \mu \mathrm{mol}$ of hydrogen peroxide per minute. The colon sample sections were mechanically homogenized on ice in a 100 mM Trizma buffer $\mathrm{pH} 7.4$ with $1 \mathrm{mM}$ EDTA and $1 \mathrm{mM}$ phenylmethanesulfonyl fluoride (PMSF). The homogenate was sonicated on ice for thirty seconds and subsequently centrifuged at $1,000 \times \mathrm{g}$ for ten minutes at $4^{\circ} \mathrm{C}$. The supernatants were then aliquoted and stored at $-80{ }^{\circ} \mathrm{C}$ until subsequent analysis. Antioxidant enzyme activities, defined as the quantity degrading $1 \mu \mathrm{mol}$ per minute of substrate (in units per mg proteins), were ascertained in triplicate using catalase (CAT), superoxide dismutase (SOD), glutathione reductase (GRed) and glutathione peroxidase (GPx) kits (Jiancheng Bioengineering Institute, Nanjing, China).

\section{Plasma antioxidant power}

A mixture containing $10 \mathrm{mM}$ ABTS (2,2'-azinobis(3-ethylbenz-thiazoline-6-sulfonic acid)) solution and $2 \mathrm{mM}$ hydrogen peroxide was stored at $4^{\circ} \mathrm{C}$ in the dark overnight. The solution was diluted to a 1:10 ratio such that an absorbance on the order of 0.31 at $660 \mathrm{~nm}$ was obtained. One milliliter (ml) of buffer of $\mathrm{pH} 3.6$, $10 \mu \mathrm{l}$ of plasma and $25 \mu \mathrm{l}$ of ABTS were added to a $10 \mathrm{ml}$ cuvette. ABTS radical cation decolorization was used to determine the total antioxidant capacity of the plasma (one of the antioxidants is ascorbic acid). The absorbance was measured five minutes after at $660 \mathrm{~nm}$. For each assay, a blank control was performed and each measurement was performed in triplicate. The results were presented as the micromolar equivalent of ascorbic acid [34]. 


\section{Cytokine measurement}

Fifty micrograms of each colon tissue sample was lysed in a cell lysis kit (Jiancheng Bioengineering Institute, Nanjing, China). In brief, the samples were first rinsed in the wash buffer and then thawed in the lysis buffer, which contained PMSF (500 mM) and a protease inhibitor cocktail. The homogenates were further treated by a freeze-thaw cycle and sonicated on ice before being centrifuged at 4,500 $\mathrm{x}$ g for four minutes. Levels of IL- $1 \beta$ and TNF- $\alpha$ in the supernatants were assayed by a specific sandwich ELISA kit (ELISA Ready-SET-GO, eBioscience, CA, USA). The amount of cytokines was normalized by the protein level of the colonic samples.

\section{Nuclear protein extraction and western blot analysis}

The extraction of nuclear and cytoplasmic proteins was performed according to the instructions of the manufacturer (Thermo Fisher Scientific, USA). For western blot analysis,each $50 \mu \mathrm{g}$ sample was separated using SDS-polyacrylamide gel electrophoresis and transferred electrophoretically to a polyvinylidene difluoride (PVDF) membrane (Bio-Rad, Hercules, CA, USA). The membrane was first blocked using evaporated milk at a $7 \%$ concentration and diluted at room temperature in Tris-buffered saline containing $0.1 \%$ Tween 20 for no less than two hours, and then incubated at $4{ }^{\circ} \mathrm{C}$ with either $\mathrm{NF}-\kappa \mathrm{B}$ (p65) or Nrf2 primary antibodies overnight. For the loading control, we used antibodies against PCNA (Sigma) and $\beta$-actin (Sigma) as the primary antibodies for the nuclear and cytoplasmic samples, respectively, and alkaline phosphatase-conjugated anti-rabbit or antimouse IgG antibodies (Promega, Madison, WI, USA) as secondary antibodies for the corresponding primary antibodies. The signals were quantified and then analyzed digitally using ImageJ (NIH), subtracting the measured intensity of each band from the background intensity. The target proteins' expression ratio was normalized against PCNA or $\beta$-actin.

\section{Statistical analysis}

We performed general statistical analysis using SPSS version 22.0, and one-way ANOVA in group comparisons to determine the homogeneity of Levene's test and Tukey's multiple comparison test. The data are expressed as the mean \pm standard error of the mean. Identical superscripts assigned to any values in a given row indicate that those values are not significantly differently at the $P>0.05$ level; different superscripts indicate significant differences between the values in a given row at the same level $(P<0.05)$.

\section{Abbreviations}

IBD: inflammatory bowel disease, MR: methionine restriction, DSS: dextran sodium sulphate, CAT: catalase, SOD: superoxide dismutase, MPO: myeloperoxidase, GPx: glutathione peroxidase, UC: ulcerative colitis, ROS: reactive oxygen species, ABTS: 2,2'-azino-bis(3ethylbenz-thiazoline-6-sulfonic acid)

\section{Author contributions}

G.L, L.Y, W.R, and Y.Y designed the experiment. G.L, L.Y, J.F, J.Y, W.R, H.N, and S.C conducted the experiment. G.L, L.Y, C.A.A, V.D, N.A.A, and Y.Y analyzed the data and prepared tables and figures. G.L, L.Y and C.A.A prepared the manuscript. All authors reviewed the manuscript.

\section{CONFLICTS OF INTEREST}

All co-authors have seen and agree with the contents of the manuscript and there is no financial interest to report.

\section{FUNDING}

This research was supported by International Partnership Program of Chinese Academy of Sciences (161343KYSB20160008), National Key Research and Development Program of China (2016YFD0500504), National Natural Science Foundation of China (No. 31330075, 31110103909, 31572416, 31402092, and 31372326), Ministry of Agricultural of the People's Republic of China (2015-Z64, 2016-X47), Hunan Provincial Science and Technology Department (2016NK2101, 2016WK2008, 2016TP2005), and Chinese Academy of Sciences visiting professorship for senior international scientists Grant no. 2016VBB007. The authors are grateful to the Deanship of Scientific Research, King Saud University for partial funding through Vice Deanship of Scientific Research Chairs.

\section{REFERENCES}

1. Molodecky NA, Soon IS, Rabi DM, Ghali WA, Ferris M, Chernoff G, Benchimol EI, Panaccione R, Ghosh S, Barkema HW, Kaplan GG. Increasing incidence and prevalence of the inflammatory bowel diseases with time, based on systematic review. Gastroenterology. 2012; 142: 46-54.e42. doi: 10.1053/j.gastro.2011.10.001.

2. Sands BE. Inflammatory bowel disease: past, present, and future. J Gastroenterol. 2007; 42: 16-25. doi: 10.1007/ s00535-006-1995-7.

3. Yin J, Ren WK, Wu XS, Yang G, Wang J, Li TJ, Ding JN, Cai LC, Su DD. Oxidative stress-mediated signaling 
pathways: A review. Journal of Food Agriculture \& Environment. 2013; 11: 132-9.

4. Ananthakrishnan AN. Epidemiology and risk factors for IBD. Nat Rev Gastroenterol Hepatol. 2015; 12: 205-17. doi: 10.1038/nrgastro.2015.34.

5. Zhu H, Li YR. Oxidative stress and redox signaling mechanisms of inflammatory bowel disease: updated experimental and clinical evidence. Exp Biol Med (Maywood). 2012; 237: 474-80. doi: 10.1258/ ebm.2011.011358.

6. Zanello G, Goethel A, Forster K, Geddes K, Philpott DJ, Croitoru K. Nod2 Activates NF-kB in CD4(+) T Cells but Its Expression Is Dispensable for T Cell-Induced Colitis. Plos One. 2013; 8. doi: ARTN e826231371/journal. pone.0082623.

7. Nishiyama Y, Kataoka T, Yamato K, Taguchi T, Yamaoka $\mathrm{K}$. Suppression of dextran sulfate sodium-induced colitis in mice by radon inhalation. Mediators Inflamm. 2012; 2012: 239617. doi: 10.1155/2012/239617.

8. McLean LP, Cross RK. Adverse events in IBD: to stop or continue immune suppressant and biologic treatment. Expert Rev Gastroenterol Hepatol. 2014; 8: 223-40. doi: 10.1586/17474124.2014.881715.

9. Mizoguchi A, Mizoguchi E. Inflammatory bowel disease, past, present and future: lessons from animal models. J Gastroenterol. 2008; 43: 1-17. doi: 10.1007/ s00535-007-2111-3.

10. Bang B, Lichtenberger LM. Methods of Inducing Inflammatory Bowel Disease in Mice. Curr Protoc Pharmacol. 2016; 72 : 558 1-42. doi: 10.1002/0471141755. ph0558s72.

11. Perse M, Cerar A. Dextran sodium sulphate colitis mouse model: traps and tricks. J Biomed Biotechnol. 2012; 2012: 718617. doi: 10.1155/2012/718617.

12. Reed KL, Fruin AB, Gower AC, Gonzales KD, Stucchi AF, Andry CD, O'Brien M, Becker JM. NF-kappaB activation precedes increases in mRNA encoding neurokinin-1 receptor, proinflammatory cytokines, and adhesion molecules in dextran sulfate sodium-induced colitis in rats. Dig Dis Sci. 2005; 50: 2366-78. doi: 10.1007/ s10620-005-3066-y.

13. Perrone CE, Mattocks DA, Jarvis-Morar M, Plummer JD, Orentreich N. Methionine restriction effects on mitochondrial biogenesis and aerobic capacity in white adipose tissue, liver, and skeletal muscle of F344 rats. Metabolism. 2010; 59: 1000-11. doi: 10.1016/j. metabol.2009.10.023.

14. Kabil H, Kabil O, Banerjee R, Harshman LG, Pletcher SD. Increased transsulfuration mediates longevity and dietary restriction in Drosophila. Proc Natl Acad Sci U S A. 2011; 108: 16831-6. doi: 10.1073/pnas.1102008108.

15. Petti AA, Crutchfield CA, Rabinowitz JD, Botstein D. Survival of starving yeast is correlated with oxidative stress response and nonrespiratory mitochondrial function.
Proceedings of the National Academy of Sciences of the United States of America. 2011; 108: E1089-E98. doi: 10.1073/pnas.1101494108.

16. Miller RA, Buehner G, Chang Y, Harper JM, Sigler R, Smith-Wheelock M. Methionine-deficient diet extends mouse lifespan, slows immune and lens aging, alters glucose, T4, IGF-I and insulin levels, and increases hepatocyte MIF levels and stress resistance. Aging Cell. 2005; 4: 119-25. doi: 10.1111/j.1474-9726.2005.00152.x.

17. Naudi A, Caro P, Jove M, Gomez J, Boada J, Ayala V, Portero-Otin M, Barja G, Pamplona R. Methionine restriction decreases endogenous oxidative molecular damage and increases mitochondrial biogenesis and uncoupling protein 4 in rat brain. Rejuvenation Research. 2007; 10: 473-83. doi: 10.1089/rej.2007.0538.

18. Sanchez-Roman I, Gomez A, Gomez J, Suarez H, Sanchez C, Naudi A, Ayala V, Portero-Otin M, Lopez-Torres M, Pamplona R, Barja G. Forty percent methionine restriction lowers DNA methylation, complex I ROS generation, and oxidative damage to mtDNA and mitochondrial proteins in rat heart. J Bioenerg Biomembr. 2011; 43: 699-708. doi: 10.1007/s10863-011-9389-9.

19. Ren W, Yin J, Wu M, Liu G, Yang G, Xion Y, Su D, Wu L, Li T, Chen S, Duan J, Yin Y, Wu G. Serum amino acids profile and the beneficial effects of L-arginine or L-glutamine supplementation in dextran sulfate sodium colitis. PLoS One. 2014; 9: e88335. doi: 10.1371/journal. pone. 0088335 .

20. Lobaton T, Vermeire S, Van Assche G, Rutgeerts P. Review article: anti-adhesion therapies for inflammatory bowel disease. Aliment Pharmacol Ther. 2014; 39: 579-94. doi: 10.1111/apt.12639.

21. Gasparetto M, Guariso G. Highlights in IBD Epidemiology and Its Natural History in the Paediatric Age. Gastroenterol Res Pract. 2013; 2013: 829040. doi: 10.1155/2013/829040.

22. Sann H, Erichsen J, Hessmann M, Pahl A, Hoffmeyer A. Efficacy of drugs used in the treatment of IBD and combinations thereof in acute DSS-induced colitis in mice. Life Sciences. 2013; 92: 708-18. doi: 10.1016/j. Ifs.2013.01.028.

23. Arab HH, Salama SA, Eid AH, Omar HA, Arafa el SA, Maghrabi IA. Camel's milk ameliorates TNBS-induced colitis in rats via downregulation of inflammatory cytokines and oxidative stress. Food Chem Toxicol. 2014; 69: 294302. doi: 10.1016/j.fct.2014.04.032.

24. Iba Y, Sugimoto Y, Kamei C, Masukawa T. Possible role of mucosal mast cells in the recovery process of colitis induced by dextran sulfate sodium in rats. Int Immunopharmacol. 2003; 3: 485-91. doi: 10.1016/S1567-5769(02)00299-0.

25. Pavlick KP, Laroux FS, Fuseler J, Wolf RE, Gray L, Hoffman J, Grisham MB. Role of reactive metabolites of oxygen and nitrogen in inflammatory bowel disease. Free Radic Biol Med. 2002; 33: 311-22. 
26. Mueller K, Blum NM, Mueller AS. Examination of the Anti-Inflammatory, Antioxidant, and XenobioticInducing Potential of Broccoli Extract and Various Essential Oils during a Mild DSS-Induced Colitis in Rats. ISRN Gastroenterol. 2013; 2013: 710856. doi: $10.1155 / 2013 / 710856$.

27. Sanchez-Fidalgo S, Cardeno A, Sanchez-Hidalgo M, Aparicio-Soto M, de la Lastra CA. Dietary extra virgin olive oil polyphenols supplementation modulates DSSinduced chronic colitis in mice. J Nutr Biochem. 2013; 24: 1401-13. doi: 10.1016/j.jnutbio.2012.11.008.

28. Beloqui A, Coco R, Alhouayek M, Solinis MA, RodriguezGascon A, Muccioli GG, Preat V. Budesonide-loaded nanostructured lipid carriers reduce inflammation in murine DSS-induced colitis. Int J Pharm. 2013; 454: 775-83. doi: 10.1016/j.ijpharm.2013.05.017.

29. Reimund JM, Wittersheim C, Dumont S, Muller CD, Baumann R, Poindron P, Duclos B. Mucosal inflammatory cytokine production by intestinal biopsies in patients with ulcerative colitis and Crohn's disease. J Clin Immunol. 1996; 16: 144-50.

30. Hyam SR, Jang SE, Jeong JJ, Joh EH, Han MJ, Kim DH. Echinocystic acid, a metabolite of lancemaside A, inhibits TNBS-induced colitis in mice. Int Immunopharmacol. 2013; 15: 433-41. doi: 10.1016/j.intimp.2012.12.017.
31. Yin FG, Liu YL, Yin YL, Kong XF, Huang RL, Li TJ, Wu GY, Hou Y. Dietary supplementation with Astragalus polysaccharide enhances ileal digestibilities and serum concentrations of amino acids in early weaned piglets. Amino Acids. 2009; 37: 263-70. doi: 10.1007/ s00726-008-0142-6.

32. Varshney J, Ooi JH, Jayarao BM, Albert I, Fisher J, Smith RL, Patterson AD, Cantorna MT. White Button Mushrooms Increase Microbial Diversity and Accelerate the Resolution of Citrobacter rodentium Infection in Mice. Journal of Nutrition. 2013; 143: 526-32.

33. Lenoir L, Rossary A, Joubert-Zakeyh J, VergnaudGauduchon J, Farges MC, Fraisse D, Texier O, Lamaison $\mathrm{JL}$, Vasson MP, Felgines C. Lemon verbena infusion consumption attenuates oxidative stress in dextran sulfate sodium-induced colitis in the rat. Dig Dis Sci. 2011; 56: 3534-45. doi: 10.1007/s10620-011-1784-x.

34. Gardi C, Bauerova K, Stringa B, Kuncirova V, Slovak L, Ponist S, Drafi F, Bezakova L, Tedesco I, Acquaviva A, Bilotto S, Russo GL. Quercetin reduced inflammation and increased antioxidant defense in rat adjuvant arthritis. Archives of Biochemistry and Biophysics. 2015; 583: $150-7$. 